\title{
Rotating Band Pion Production Targets for Muon Colliders and Neutrino Factories ${ }^{a}$
}

\author{
B.J. King \\ Brookhaven National Laboratory, Building 901A, P.O. Box 5000, Upton, NY11973 \\ email: bking@bnl.gov \\ web page: http://pubweb.bnl.gov/people/bking
}

\begin{abstract}
An update is presented on a conceptual design for a pion production target station using a rotating cupronickel band and that was originally proposed for use at a muon collider facility with a $4 \mathrm{MW}$ pulsed proton beam. After reviewing the salient design features and motivations for this target, ongoing studies are described that are attempting to benchmark the thermal stresses and radiation damage on the target band using data from the Fermilab antiproton source and other operating targets. Possible parameter optimizations and alternative technologies for the rotating band are surveyed, including discussion on the the various proton beam parameters that might be encountered for rotating band targets at either muon colliders or neutrino factories. Finally, an outline is proposed for a possible R\&D path towards capability for the actual construction of rotating band pion production targets.
\end{abstract}

\section{Introduction and Motivation}

Conceptual design studies for muon colliders that have taken place since the mid1990's have motivated the design of pion production targets that can operate and survive with megawatt-scale pulsed proton beams. Over the past few months, the design timescale and potential learning curve for such targets has effectively been abbreviated by the expanded interest in neutrino factories - muon storage rings dedicated to producing neutrino beams that will require similarly large muon currents to muon colliders and that have prospects for being built on a shorter timescale perhaps to be ready within the next decade.

We present an update on a previous report [1] describing a conceptual design for a cupronickel rotating band pion production target for muon colliders that was proposed as a relatively conservative extrapolation from existing targets. A more detailed write-up on this target is in progress [2]. (Note that another rotating band design based on reference [1] is presented elsewhere in these proceedings [3].)

The rotating band design is readily adaptable to neutrino factories and, indeed, its conservative nature makes its development particularly well matched to the shorter timescales for this application. Alternative, more exotic, targetry options that were originally proposed for muon colliders - such as pulsed mercury jets may involve extensive multi-year exploratory experimental R\&D programs 顿 that do not appear to be particularly compatible with the shorter timescales envisaged for neutrino factories.

This paper is laid out as follows. General design considerations and strategies for high power solid targets form the topic of the following section. Sections 3 through section 5 then focus in on a review of the specific cupronickel band design that was proposed in reference [1]. Specifically, section 3 gives an overview of the

${ }^{a}$ Presented at the ICFA/ECFA Workshop "Neutrino Factories based on Muon Storage Rings" ( $\nu$ FACT'99), Lyon, France, 5-9 July, 1999. 
conceptual design and strawman specifications, section 1 summarizes the results of computer simulations predicting its pion yield performance and characterizing the heating effects from the beam, and section 5 discusses stress and durability issues. Section 6 steps back from the parameters in [1] to examine the technology options and parameter optimizations available to the rotating band target concept for the spectrum of possible proton driver scenarios at both muon colliders and neutrino factories. The paper concludes with comments on the potential for the rotating band target concept and with an outlook on the R\&D program required to bring this conceptual design to practical fruition.

\section{General Design Strategies for High Power Production Targets}

This section gives an overview of the general design goals that were considered important when proposing the target design of reference [1], and on the strategies employed to achieve these design goals. The intention was to design a target station that:

1. can be designed quickly and with relatively modest resources, so it will assuredly not hold up the overall development of neutrino factories or muon colliders

2. will clearly survive any beam-induced stresses it might be subjected to and have an acceptable lifetime

3. is relatively straightforward and affordable to build and maintain (including target replacement and disposal)

4. has pion yields per proton and phase space densities that are as good as, or not much inferior to, what could be achieved with the more idealized targets that could be designed for operating with low beam powers.

The first item implies that the R\&D program for such a target can be conducted mainly through paper studies, computer simulations and engineering computerassisted design studies, perhaps augmented by a modest amount of mechanical prototyping and/or beam tests if convenient. Such an R\&D program appears plausible for the strawman design scenario of reference [1]. Items 3 and 4 also appear to be met by this scenario, as can be judged from the information presented in section 3 of this paper and elsewhere [1, 2].

The remainder of this section addresses item 2 in the list, since the single most difficult design constraint for rotating bands and other solid targets for muon colliders is the requirement of survivability in the face of instantaneous beam energies per proton pulse of order $100 \mathrm{~kJ}$ and megawatt-scale beam powers, i.e., comparable to or larger than the largest existing proton facilities. A sound design strategy to satisfy item 2 is to choose beam and target parameters such that the maximum material stresses and radiation exposures do not go beyond what has been explicitly achieved in existing targets or targetry studies. It is clear that this can always be achieved in principle, even for the highest beam powers under consideration, by: 
1. choosing a target material with appropriate mechanical properties and moderate thermal stresses, consistent with optimizing the pion yield. In practice this might mean choosing from materials with medium atomic numbers in the range from titanium $(Z=22)$ through nickel $(Z=28)$, as discussed further in sections 4 and 6 , and

2. sufficiently spreading out the spot size to cope with higher energy beam pulses, and

3. rotating or otherwise moving the target to continually expose new areas of the target to each beam pulse. This limits both the instantaneous local thermal stresses and the lifetime radiation exposure of the target material.

Concerning the final item of strategy, the considerable potential for designs that continually expose the beam to new target material is amply illustrated by the tungsten target design for the proposed Accelerator Production of Tritium (APT) project [5] at Los Alamos National Laboratory. A $1.7 \mathrm{GeV}, 100 \mathrm{~mA}$ beam is continually rastered across a $19 \times 190 \mathrm{~cm}$ area of the APT tungsten target to limit the local heating and thermal shock stresses. Impressively, the projected $170 \mathrm{MW}$ beam power onto the APT target is approximately two orders of magnitude larger than the applications considered in this paper.

As a detailed difference from the approach for the APT, the band target uses the more traditional solution for high power targets of moving the target material rather than the beam spot. The idea of rotating or scanning high-power targets has already found successful application in several existing facilities including, for example, the trolled tungsten-rhenium target for the SLC positron source [6] and the rotating nickel target at the BNL "g-minus-2" experiment [7]. Target rotation can limit temperature rises and the consequent thermal stresses on timescales of a second or less and, in the longer term, it spreads out the radiation load over much more material. The Fermilab antiproton source target [8] is rotated more slowly than the preceding examples in order to spread out the radiation dose over the circumference of the target disk, as is discussed further in section 5 .

\section{A Conceptual Design and Straw-man Specifications for a Cupronickel Rotating Band Target}

Table 1: Strawman parameters for the cupronickel target band, taken from reference 11.

\begin{tabular}{|r|c|}
\hline target band radius $(\mathrm{R})$ & $2.5 \mathrm{~m}$ \\
band thickness $(\mathrm{t})$ & $0.6 \mathrm{~cm}$ \\
band width $(\mathrm{w})$ & $6 \mathrm{~cm}$ \\
beam path length in band $(\mathrm{L})$ & $30 \mathrm{~cm}$ \\
proton interaction lengths $(\lambda)$ & 2 \\
band tilt angle $(\alpha)$ & $150 \mathrm{mrad}$ \\
band rotation velocity $(\mathrm{v})$ & $3 \mathrm{~m} / \mathrm{s}$ \\
\hline
\end{tabular}




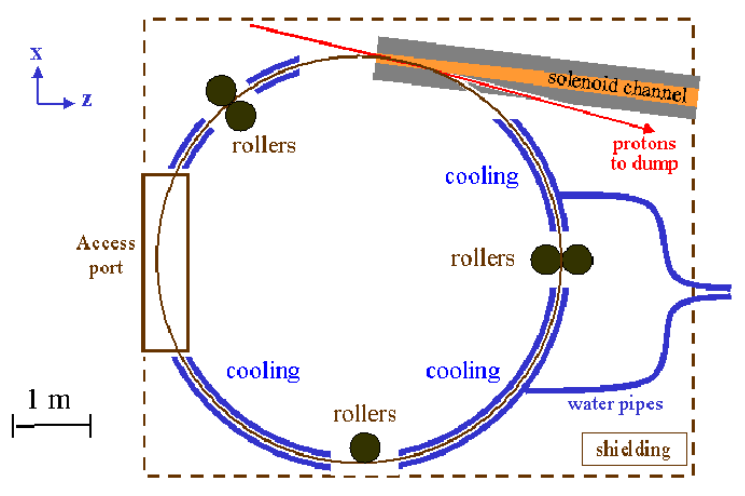

Figure 1: A conceptual illustration of the targetry setup proposed in reference 1 .

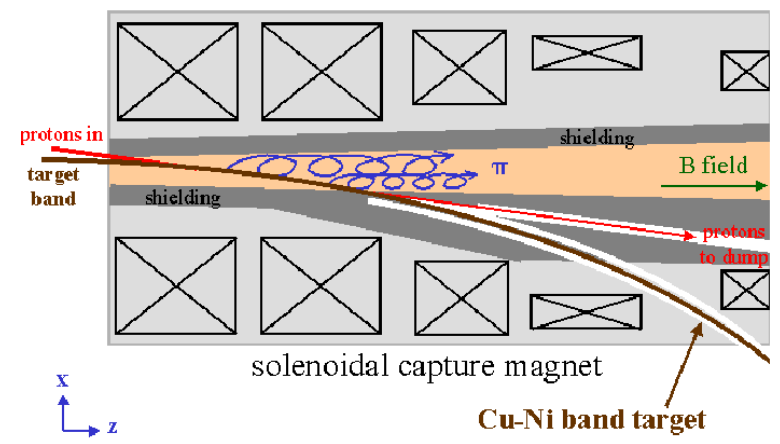

Figure 2: A conceptual illustration [1] of the target layout around the pion production region.

Table 2: The proton beam parameters that correspond to the strawman target parameters of table 1. Except for the beam spot size, which is specific to the target design, these parameters have been taken from reference 9 and are appropriate for the muon collider specifications in that reference.

\begin{tabular}{|l|l|}
\hline parameter & value \\
\hline beam energy & $16 \mathrm{GeV}$ \\
protons per pulse $(\mathrm{ppp})$ & $1.0 \times 10^{14}$ \\
pulse energy & $256 \mathrm{~kJ}$ \\
pulse duration & instantaneous $(<<1 \mu \mathrm{sec})$ \\
pulse repetition rate & $15 \mathrm{~Hz}$ \\
beam power & $3.84 \mathrm{MW}$ \\
gaussian spot size & $\sigma_{x}=1.5 \mathrm{~mm}, \sigma_{y}=10 \mathrm{~mm}$ \\
\hline
\end{tabular}




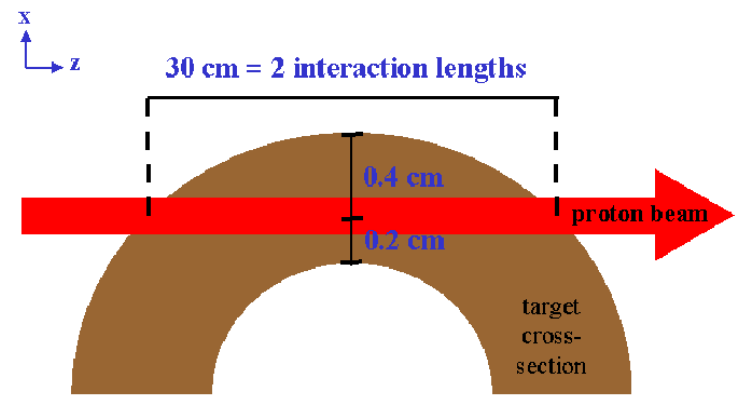

Figure 3: The trajectory of the proton beam into the cupronickel target band [1]. The aspect ratio is very distorted - only a small chord of the target circumference is shown.

The discussion presented in this section and the two that follow are specific to the cupronickel rotating band target design proposed in reference [1], beginning with the overview of that conceptual design given in this section.

Figure 1 gives a schematic overview of the target concept presented in reference [1] and figure 2 zooms in on the production region. It should be emphasized that such details as the rollers and cooling setup are shown only schematically and that no concerted effort has been put into their design or layout. The cupronickel target band is enclosed in a 20 Tesla solenoidal magnetic pion capture magnet whose general design has previously been studied [9] by the Muon Collider Collaboration. The pion secondaries spiral along the solenoidal magnetic channel before decaying into the muon bunches needed for cooling, acceleration and injection into the collider ring. The radius of the solenoidal channel, $7.5 \mathrm{~cm}$, and the magnetic field strength, 20 Tesla, are those commonly assumed for recent muon collider studies [9]. The design modification specific to this particular geometry concerns the provision of entry and exit ports for the target band. Example coil geometries [10] show that these ports can be provided with little modification to the design of the solenoidal channel.

Table 1 gives some relevant parameters for the cupronickel band and figure 3 illustrates the trajectory of the proton beam into the target band. The band parameters correspond to the proton beam parameters for a muon collider that are given in table 2. Different parameter values might be appropriate for alternative beam scenarios at muon colliders or for neutrino factories. The geometry of the band is chosen to approximately maximize the pion yield. The general requirements are that the proton pathlength through the target material should be approximately 2 nuclear interaction lengths and that the band should be thin enough to allow most of the pions to escape the target. To optimize the pion yield [1], the trajectory of the beam through a chord of the target band is at a tilt angle of 150 milliradians to the axis of the solenoid. 
The cupronickel band is guided and powered by several sets of rollers that can be connected by driveshafts to remotely housed motors outside any radiation shielding. This scenario has been taken from the Zenzimmer mills that are used for pressing metal sheets and has the attraction of being mechanically very simple in the high radiation area surrounding the production region. Bennett [3] even suggests the total elimination of moving parts other than the target band by using electromagnetic guidance and rotation of the target band by linear motors. Procedures for installation and extraction of the target band are proposed in reference [2].

The rotation rate of $3 \mathrm{~m} / \mathrm{s}$ given in table 1 corresponds, for the $15 \mathrm{~Hz}$ beam frequency, to a target advance per pulse by $1 / 3$ of the chord spanned by the proton beam. The three overlapping proton pulses in any part of the band imply [1] a maximum total temperature rise approximately double the instantaneous rise from each individual pulse. Temperature rises and stresses will be further discussed in section 5 .

A competing concern to the target heating stress that limits the acceptable rotation rate of the target is the eddy currents induced by the rapidly rotating band in a strong magnetic field. The eddy current power is proportional to the conductivity and to the square of the band velocity and a very approximate analytic calculation [2] predicts that the power dissipated will be of order several $\mathrm{kW}$. This is more than an order of magnitude below the beam heating and so is clearly a manageable heat source. The power will have to be supplied by the electric motor driving the rotation of the target and the requirements on the drive mechanism are within typical operating parameters for Zenzimmer steel mills.

Cupronickel alloys are preferred over both copper and nickel for the particular targetry application considered in this paper because their lower electrical conductivity will reduce the eddy currents from rotation through the magnetic field of the solenoidal capture magnet. Cupronickel alloys such as, for example, alloy 715 produced by Olin Brass [1] have essentially the same density and interaction lengths as copper and nickel and have very similar mechanical properties. However, Olin alloy 715 has an electrical conductivity at 20 degrees centigrade of only $2.6 \mathrm{MS} / \mathrm{m}$, compared to $58 \mathrm{MS} / \mathrm{m}$ for copper and $14 \mathrm{MS} / \mathrm{m}$ for nickel.

The hot portion of the band is rapidly carried away from the production region into a water cooling channel, as is the case for the nickel production target at the BNL g-2 experiment [7]. Although both the peak temperature and power are much larger than in the g-2 experiment, the surface area of the band has been chosen large enough to give heat transfer rates of approximately $30 \mathrm{~W} / \mathrm{cm}^{2}$ [1] that are comfortable even in the presence of a steam boundary layer. It has been suggested [3 that the target and capture channel should be in a helium atmosphere to allow the easy distillation of water vapor and any other impurities. This will have negligible effect [2] on pion production due to helium's low density and low atomic number. 


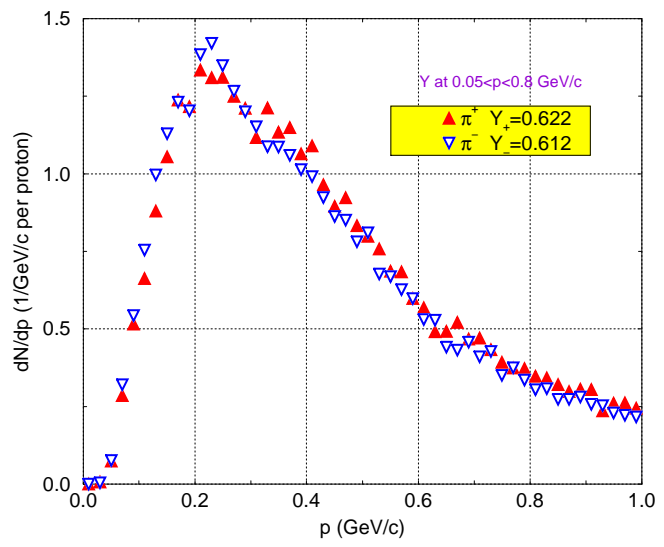

Figure 4: Pion momentum spectra [1] at the plane $90 \mathrm{~cm}$ downstream from the central intersection of the beam with the target, determined for the beam and target parameters of tables 2 and 1 . respectively.

Table 3: A summary of the MARS Monte Carlo results and derivative predictions for pion yields and thermal parameters that were presented in reference 11 . The simulation used the target band and proton beam parameters of tables 1 and 2 , corresponding to a $3.84 \mathrm{MW}$ proton beam power.

\begin{tabular}{|r|c|}
\hline parameter & $\mathrm{CuNi}$ \\
\hline$\pi^{+}\left(\pi^{-}\right)$yield/proton & $0.622(0.612)$ \\
peak energy deposition/pulse & $69 \mathrm{~J} / \mathrm{g}$ \\
peak inst. temperature rise & $151^{\circ} \mathrm{C}$ \\
peak total temperature rise & $\sim 300^{\circ} \mathrm{C}$ \\
total power deposition in target band & $0.324 \mathrm{MW}$ \\
average cooling rate & $31 \mathrm{~W} \cdot \mathrm{cm}^{-2}$ \\
\hline
\end{tabular}

\section{Pion Yield Predictions and Thermal Parameters of the Cupronickel Band Target}

Detailed MARS [12] tracking and showering Monte Carlo simulations were performed [1] to obtain the pion yields per proton for the beam and target parameters of [1] that are reproduced in tables 1 and 2. The graphical results in figure 4 correspond to yields of $Y_{+}=0.622$ and $Y_{-}=0.612$ positive and negative pions per proton for the momentum range $0.05<\mathrm{p}<0.80 \mathrm{GeV} / \mathrm{c}$. The peak energy deposition density was found to be $68.6 \mathrm{~J} / \mathrm{g}$ per pulse, corresponding to a temperature rise of $\Delta T=151^{\circ} \mathrm{C}$ and a total power dissipation in the target of $0.324 \mathrm{MW}$. These predictions are summarized in table 3 .

These pion yields and the predicted phase space densities are almost identical to the best predicted yields for the exotic liquid mercury jet targets that are also under consideration 泊 for muon colliders and neutrino factories. Optimization studies [13, 9] for both mercury jet and band targets further suggest that such 
yields are rather close to the optimum that could be obtained even for a low power proton beam, thus satisfying the fourth of the design goals stated in section 2 .

\section{$5 \quad$ Stress and Durability Issues for the Cupronickel Band}

As an application of the design strategy presented in section 2, this section examines the possibilities for evaluating the survivability of the cupronickel band target through benchmarking to existing targets. The Fermilab antiproton source target [8] appears to be one of the most suitable targets for benchmarking the band, so we begin by summarizing its design and operating parameters.

The Fermilab antiproton target consists of [14] a vertical stack of 3 nickel target disks plus one copper target disk, each approximately $1 \mathrm{~cm}$ thick and $4.7 \mathrm{~cm}$ in radius, and interspersed with copper cooling disks. The target is cooled by forcing air up the vertical axis and through channels in the copper cooling disks. The stack of disks is enclosed in a titanium can that would contain target the material in case of failure, but the can is not in contact with the disks and so is irrelevant for considerations of mechanical survivability. A $120 \mathrm{GeV}$ proton beam passes through a chord of the selected target disk, with an intensity [B] of 1.6 to $2.1 \times 10^{12}$ protons per $1.6 \mu$ sec pulse, which is incident every 2.4 seconds.

The energy per pulse of the antiproton source, up to $40 \mathrm{~kJ}$, is almost an order of magnitude below the $256 \mathrm{~kJ}$ muon collider specification of table 2. Despite this, the round beam spot has a gaussian sigma at entry of only 140 microns [8], and this exposes the antiproton target to local energy deposits and temperature rises much larger than MARS predictions for the cupronickel band, which assume a much larger elliptical spot of dimensions $\sigma_{x}=1.5 \mathrm{~mm}, \sigma_{y}=10 \mathrm{~mm}$. As shown in table 1, the cupronickel band is predicted to sustain a maximum instantaneous energy deposition of approximately $70 \mathrm{~J} / \mathrm{g}$ per proton pulse, compared to the 500-600 J/g maximum depositions at the the Fermilab antiproton source. The impressive peak temperature rise in the antiproton target is $1100^{\circ} \mathrm{C}$ over $1.6 \mu \mathrm{sec}$, to be compared to $150^{\circ} \mathrm{C}$ instantaneously and approximately $300^{\circ} \mathrm{C}$ over a fraction of a second for the band.

While the comparison of the preceding paragraph is suggestive that the instantaneous heat stresses on the cupronickel band might be acceptable, there are several issues to be resolved before one has confidence in the benchmarking comparison with the Fermilab antiproton target. Issues include the effects of the different target geometries and the question of how closely the $1.6 \mu \mathrm{sec}$ timescale for energy deposition in the antiproton target approximates the instantaneous energy deposition in the band.

To further understand the shock heating stresses on the cupronickel band, finite element computer simulations have been performed [15] using ANSYS, a commercial package that is very widely used for stress and thermal calculations. Energy density distributions for the simulations were generated using the MARS [12] particle production Monte Carlo package. The very preliminary simulations show periodic returns to maximum stress (i.e. "ringing") but with little or no amplification beyond the initial stress. This is encouraging, and seems plausible given that the oscillations occur in the band dimension that is much shorter than the other two so 
the geometry is quasi-one dimensional and with little potential for focusing. Further ANSYS simulations of band target geometries are commencing [16], and these are intended to include explicit benchmarking simulations on the Fermilab antiproton target.

The other threat to the survival of the target band comes from exposure to radiation. This can change the material properties of target materials by transmuting some of the target atoms to new isotopes or elements and by causing dislocations in the atomic lattice. Face-centered cubic metal lattices such as copper and nickel are known from experience to survive radiation damage better than body-centered cubic metals like iron or tungsten. Target damage studies at Los Alamos National Laboratory and elsewhere 117 predict a risk of failure for copper and nickel targets after integrated doses somewhere in the range $10^{21}$ to $10^{22}$ minimum ionizing particles per square centimeter, which corresponds to approximately $0.3-3 \mathrm{GJ} / \mathrm{g}$ of deposited energy.

It is straightforward to obtain a rough estimate of the integrated doses on the cupronickel by noting that the parameters of tables 1 and 2 correspond to doses, along the centerline of the band and for an accelerator year of $10^{7}$ seconds, that accumulate to:

$$
\text { summed energy deposition } \sim 140 \mathrm{~J} / \mathrm{g} \times \frac{3 \mathrm{~m} / \mathrm{s}}{2 \pi \times 2.5 \mathrm{~m}} \times 10^{7} \mathrm{~s} \sim 0.3 \mathrm{~J} / \mathrm{g},
$$

where $140 \mathrm{~J} / \mathrm{g}$ is the energy deposited per rotation and the second term is the target rotation frequency. Since $0.3 \mathrm{GJ} / \mathrm{g}$ is the lower limit for predictions of target failure, the initial conclusion to be drawn is that it may well be acceptable to replace the target band after each year's running. More detailed studies are obviously needed to check and refine this first simple estimate.

To recap, this section has provided a first look at benchmarking the cupronickel band target to the Fermilab antiproton target and other existing data from operating targets. The two indications from these initial comparisons are that:

1. the much greater temperature rises in the operating Fermilab target give some initial confidence in the short-term survivability of the target but the comparison has not yet been made rigorous

2. the simple calculation of equation 1 suggests that potentially damaging radiation doses would accumulate over an acceptably long timescale for the cupronickel band target, despite the $3.8 \mathrm{MW}$ beam power, because the radiation dose is spread over the entire circumference of the band rather than being concentrated in one region.

More detailed studies are beginning to check and clarify these very preliminary findings.

\section{Technology Options and Parameter Optimizations}

The parameters of the cupronickel band target presented in reference [1] and section 3 represent no more than an educated guess at a relatively optimal configuration 
for the $4 \mathrm{MW}$ proton driver parameters of table 2. The R\&D required to verify and refine this design is just beginning and it is expected that other parameter values and design refinements will likely turn out to be better suited for this and other proton driver scenarios.

Entry-level neutrino factories have been discussed with proton driver powers of $1 \mathrm{MW}$ or less, and this will clearly allow some relaxation of target design parameters. At the other end of the scale, proton drivers for neutrino factories of up to $20 \mathrm{MW}$ have also been discussed at CERN. In some neutrino factory scenarios, the proton beam is partitioned into smaller bunches than is feasible for muon colliders and this will generally also allow for a relaxation of target parameters.

Some examples of parameters that need to be optimized depending on the specific proton driver scenario are:

1. the beam spot size and the cross-sectional area of the band. These will tend to become larger for increasing proton pulse energy

2. the target circumference and rotation rate: these will both tend to increase with increasing average beam power; the first to increase the surface area available for cooling the target and the second to moderate the localized target heating.

These parameter values and the those of the proton driver will also determine the optimal technology decisions for several design options, including:

1. the cooling technology. Helium gas cooling might perhaps be technically simpler than water cooling but the latter can provide larger heat transfer rates per unit area. Radiative cooling is a third possible option for refractory target materials such as graphite [18], tantalum [3] or tungsten

2. the band drive and guidance. The optimal choice might depend on the level of frictional drag from eddy currents in the magnetic field of the capture solenoid and this depends in turn on the target band cross section, the target material and the target rotation velocity. The Zenzimmer-type rollers presented in reference [1] can comfortably handle the several kilowatt frictional drag for the cupronickel band and default parameter set. Smaller frictional loads would instead allow for more modest electromagnetic guidance and a linear electric drive, as suggested in reference [3]

3. the choice of target band material, as will now be discussed further.

Evaluations of several targets for pion yield and for heating and shock stresses were performed [13, 18] using MARS simulations. It should be noted that these comparisons between elements depended on target densities and on the specific targetry scenario used, so they provide only approximate guidance. However, the trend in heating stress was clear, with the stresses best for low atomic number (Z) elements and becoming rapidly worse with increasing $Z$. To balance this, pion yields were predicted to be lower for low- $\mathrm{Z}$ materials than for those with medium or high atomic number. Instead of a steady rise in yield, the yield was found to plateau somewhere between $\mathrm{Al}(\mathrm{Z}=13)$ and $\mathrm{Cu}(\mathrm{Z}=29)$ (elements in between were 
not investigated) and then to remain constant to within the accuracy of the study all the way out to the high- $Z$ elements tungsten $(Z=74)$ and mercury $(Z=80)$.

This comparative study of target elements, along with the outstanding track record of both copper and nickel as target materials, was part of the basis for the choice of cupronickel as the target band material proposed in reference [1], with nickel $(Z=27)$ and copper $(Z=28)$ both towards the low- $Z$ end of the plateau in pion yield. It would clearly be helpful to repeat the yield study for elements between $\mathrm{Al}$ and $\mathrm{Cu}$ to pin down the exact fall-off position of the yield plateau, especially since some of the elements in between and their alloys are known to have excellent mechanical and thermal properties for targetry applications, particularly $\mathrm{Ti}(\mathrm{Z}=22)$, $\mathrm{V}(\mathrm{Z}=23), \mathrm{Cr}(\mathrm{Z}=24)$ and $\mathrm{Mn}(\mathrm{Z}=25)$ 19, 20].

While initial studies suggest the suitability of these medium- $Z$ elements for a $4 \mathrm{MW}$ proton driver for muon colliders, lower-Z elements such as graphite can always be considered as options to extend the rotating band design to larger safety margins or to even more demanding beam specifications. The penalty to be paid is that graphite, for example, appears to have [21] only about $2 / 3$ the pion yield of the elements on the yield plateau. Despite this, such bands might anyway be used in the same target station as uses medium-Z bands - either as insurance against problems with the medium- $Z$ band or as an entry-level band to be eventually replaced by one with a higher yield.

\section{Conclusions and Outlook}

This paper has reviewed and enlarged on a previously proposed design [1] for a high power pion production target station based around a rotating cupronickel band target. The design scenario is mechanically rather straightforward and can be readily extrapolated from, and benchmarked to, existing targets. Initial computer simulations [1] have predicted relatively optimal pion yields and the initial comparisons, made in section 5 , with the operating Fermilab antiproton target suggest that the cupronickel band target parameters of [1] will survive the proton beams for at least some of the neutrino factory and muon collider scenarios that are under discussion. More generally, it was argued in sections 2 and 6 that viable rotating band target designs almost certainly exist for any of the proton driver scenarios that have been seriously discussed for either muon colliders or neutrino factories. The question is one of design optimization rather than feasibility, and whether the demands of target survivability force any significant compromises on the pion yield or phase space density that can be supplied by the target.

The evolutionary nature and relative simplicity of the target design and concept appears to make it compatible with a few-year R\&D program to first explore the options and parameter space and then, if all goes well, to refine and develop the design towards construction at a neutrino factory or muon collider. Such an R\&D program might develop something like this:

- in 2000: A) Paper studies to further clarify the design options and issues, including developing databases of target material properties, existing targets, past experimental targetry studies and contact information on targetry experts and contacts, B) stress simulations and optimization studies using AN- 
SYS or a similar finite element analysis package. C) beginning engineering studies on the target layout, mechanical issues and cooling options, D) develop a conceptual design for the beam dump, E) further yield optimization studies on target geometry and the band material, using MARS or similar particle production codes, F) further particle tracking studies to explore the integration of the target design with the beam dump and capture and phase rotation channels.

- in 2001: More detailed design studies for specific scenarios. Detailed assessments of, and comparisons with, other target options.

- 2002 and beyond: continuing design studies might lead to mechanical prototyping if this is found to be necessary and to beam tests if these are convenient and would add significantly to the existing pool of experimental knowledge.

- by approximately 2004: ready to begin constructing a rotating band target for a neutrino factory or muon collider.

The R\&D issues for successive years have been spelled out in progressively less detail but will necessarily involve a ramp-up in manpower, beginning from perhaps 2 full-time equivalent people in 2000. Such an R\&D program looks to be of modest extent compared to the efforts required for the more exotic targets needed for, e.g., neutron spallation sources. Indeed, the overall program looks compatible with installing a rotating band target station at a neutrino factory or muon collider facility for potential operation as early as 2006 or 2007.

\section{Acknowledgments}

Studies on the cupronickel band target design have been conducted in collaboration with R.J. Weggel, N.V. Mokhov and S.S. Moser. This work has also benefitted from discussions and techical advice from G. Bunce and C. Pai. The organizers and secretariat of NUFACT99 are to be commended for a well-organized and stimulating workshop.

This work was performed under the auspices of the U.S. Department of Energy under contract no. DE-AC02-98CH10886.

\section{References}

[1] B.J. King, S.S. Moser, R.J. Weggel and N.V. Mokhov, "A Cupronickel Rotating Band Pion Production Target for Muon Colliders", Proc. PAC'99, BNL66236 .

[2] B.J. King, S.S. Moser, R.J. Weggel and N.V. Mokhov, paper in preparation.

[3] Private communication with J.R.J. Bennett (RAL).

[4] Alessi et al., "An R \& D Program for Targetry and Capture at a MuonCollider Source - A Proposal to the BNL AGS Division". Now accepted into 
the BNL experimental program as experiment E951, with spokesperson Kirk T. McDonald (Princeton U.).

[5] The web page for the Accelerator Production of Tritium (APT) project is http://apt.lanl.gov/. The APT target layout is illustrated and the basic overall design parameters are tabulated.

[6] E. Reuter et al., "Mechanical Design and Development of a High Power Target System for the SLC Positron Source" IEEE PAC (1991).

[7] Internal notes on the BNL g - 2 pion production target, courtesy G. Bunce. Private communications with G. Bunce and C. Pai (BNL).

[8] S. O'Day et al., "New Target Results from the FNAL Antiproton Source", Proc. IEEE 1993 PAC 4, 3096.

[9] C. Ankenbrandt et al. (The Muon Collider Collaboration), "Status of Muon Collider Research and Development and Future Plans", Phys. Rev. ST Accel. Beams 2, 081001 (1999).

[10] R.J. Weggel, unpublished plots of magnet coil configuration and on-axis magnetic field profile. These plots can be viewed at

http : //pubweb.bnl.gov/people/bking/target ${ }_{b}$ erkeley99/sld010.htm and

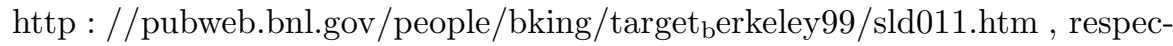
tively.

[11] Olin Brass, http : //www.olinbrass.com/

[12] N.V Mokhov, "The MARS Code System User's Guide, Version 13 (98)", FERMILAB-FN-628 (Feb. 1998).

[13] The $\mu^{+} \mu^{-}$Collider Collaboration, $\mu^{+} \mu^{-}$Collider Feasibility Study, BNL52503, FERMILAB-Conf-96/092, LBNL-38946 (July 1996);

http://www.cap.bnl.gov/mumu/book.htm

[14] S. O'Day (FNAL) unpublished engineering drawing of the Fermilab antiproton source target, dated 1-31-94.

[15] Private communications with S.S. Moser (St. Joseph's College, Indiana).

[16] Private communications with C.J. Densham and R.J.R. Bennett (RAL).

[17] Private communications with N.V. Mokhov (FNAL).

[18] "FNAL Feasibility Study on a Neutrino Source Based on a Muon Storage Ring", report in preparation, http://www.fnal.gov/projects/muon_collider/nu-factory/.

[19] "Conceptual Design of a $500 \mathrm{GeV}$ e+e- Linear Collider with Integrated X-ray Laser Facility". DESY 1997-048, ECFA 197-182 (1997). See table 3.4.4, p. 429 for the parameters of various titanium alloys that are candidates for high power production targets. 
[20] An archive of material properties relevant to fusion reactors, assembled by the ARIES Fusion Power Plant Studies Team, can be found at http://aries.ucsd.edu/PROPS/props.html.

[21] N.V. Mokhov, "Pi/Mu Yield and Power Dissipation for Carbon and Mercury Targets in 20-Tesla Solenoid with Matching Section", Muon Collider Internal Note 61, (1999). 